\title{
Characteristics of Newborns Born to SARS-CoV-2- Positive Mothers: A Retrospective Cohort Study
}

\author{
Mohsen A.A. Farghaly, MD ${ }^{1,2}$ Fernanda Kupferman, MD ${ }^{1}$ Fiorella Castillo, MD, MSPH ${ }^{10}$ \\ Roger M. Kim, MD, MPH ${ }^{1}$ \\ ${ }^{1}$ Department of Pediatrics, Brookdale University Hospital Medical \\ Center, Brooklyn, New York \\ ${ }^{2}$ Department of Pediatrics, Aswan College of Medicine, Aswan, Egypt \\ Address for correspondence Fiorella Castillo, MD, MSPH, Department \\ of Pediatrics, Brookdale University Hospital Medical Center, Brooklyn, \\ NY 11212 (e-mail: ficastil@bhmcny.org).
}

Am J Perinatol 2020;37:1310-1316.

\begin{abstract}
Keywords

- COVID-19

- newborn

- NICU

- pandemic

- SARS-CoV-2

Objective The novel virus known as severe acute respiratory syndrome-coronavirus-2 (SARS-CoV-2) has led to a terrifying pandemic. The range of illness severity among children is variable. This study aims to assess the characteristics of newborns born to SARS-CoV-2-positive women compared with those mothers who tested negative.

Study Design This was a retrospective cohort study performed at Brookdale Hospital Medical Center in New York City from March to May 2020. Electronic medical records of mother-baby dyads were reviewed.

Results Seventy-nine mothers tested for SARS-CoV-2 were included, out of which $18.98 \%$ of mothers tested SARS-CoV-2 positive. We found a significant association between symptoms and SARS-CoV-2 status. We observed a significant association between newborns of SARS-CoV-2 positive and SARS-CoV-2 negative mothers regarding skin-to-skin contact $(p<0.001)$. Both groups showed significant differences regarding isolation $(p<0.001)$. Interestingly, regarding SARS-CoV-2 infection in newborns, only one newborn tested SARS-CoV-2 positive and was unstable in the neonatal intensive care unit (NICU). With the multivariable logistic regression model, babies of SARS-CoV-2 positive mothers were three times as likely to have desaturations in comparison to newborns from negative mothers. Also, newborns of SARS-CoV-2positive mothers were four times more likely to have poor feeding, compared with newborns of SARS-CoV-2-negative mothers. Finally, babies of SARS-CoV-2-positive mothers were ten times more likely to be symptomatic at the 2-week follow-up.

Conclusion SARS-CoV-2 has caused major morbidity and mortality worldwide. Neonates born to mothers with confirmed or suspected SARS-CoV-2 are most of the time asymptomatic. However, neonatal critical illness due to SARS-CoV-2 is still a possibility; thus, isolation precautions (such as avoiding skin-to-skin contact and direct breastfeeding) and vertical transmission should be studied thoroughly. In addition, testing these newborns by nasopharyngeal swab at least at 24 hours after birth and monitoring them for the development of symptoms for 14 days after birth is needed.
\end{abstract}

received

July 7,2020

accepted after revision

July 30,2020

published online

September 3, 2020
Copyright $\odot 2020$ by Thieme Medical Publishers, Inc., 333 Seventh Avenue, New York, NY 10001, USA Tel: $+1(212) 760-0888$
DOI https://doi.org/ 10.1055/s-0040-1715862. ISSN 0735-1631. 


\section{Key Points}

- For SARS-CoV-2-positive mothers, reducing transmission of infection to newborns is crucial.

- Newborns of SARS-CoV-2-positive mothers are usually asymptomatic and may not be easily infected.

- Critical illness in the newborn may still happen, so monitoring is needed.

The novel virus known as severe acute respiratory syndromecoronavirus-2 (SARS-CoV-2) has led to the global pandemic, novel coronavirus disease 2019 (COVID-19). ${ }^{1,2}$ Denoted as the epicenter of the pandemic, New York City has been hit hard by SARS-CoV-2 infections, tragically overwhelming the city's hospital capacities. The range of illness severity among pregnant women with SARS-CoV-2 is variable. ${ }^{3}$ Little is known about perinatal transmission and neonatal infection by the SARS-CoV-2 virus, as there is limited data about a possible vertical transmission. ${ }^{4}$

Disease characteristics among newborn patients in the United States have not been described. ${ }^{5}$ In addition, there is limited data regarding the benefits and risks of maternal screening for SARS-CoV-2, and/or taking special precautions during the deliveries, use of neonatal resuscitation including airway management, skin-to-skin contact, infant placement/ isolation, neonatal testing, newborn's type of feeding, and visiting policies. ${ }^{6}$ As the number of SARS-CoV-2 cases continues to increase in the United States, it will be important to gather data to adapt screening strategies to reduce the overburdening of health departments. ${ }^{5}$

Brookdale Hospital Medical Center (BHMC) is one of the hospitals that overwhelmed by the disease burden. Since March 2020, all pregnant women admitted for delivery to BHMC were screened for SARS-CoV-2. Of those who tested positive, we screened their babies for SARS-CoV-2 within the first 24 hours of life, at 48 hours, and at 1 week of age. We also followed them clinically for the development of any symptoms for 2 weeks.

\section{Materials and Methods}

This was a retrospective cohort study performed in the neonatal intensive care unit (NICU) and Mother-Baby Unit (also known as Nursery) at BHMC in New York City from March to May 2020.

Electronic medical records of mother-baby dyads were reviewed. Data on maternal sociodemographic factors (age, race, smoking, alcohol, or drug use), comorbidities, contact to suspected or confirmed SARS-CoV-2 case, any symptoms of SARS-CoV-2, presence or absence of chorioamnionitis, review of clinical examination of the obstetricians and midwives, review of antenatal laboratory evaluations, and SARSCoV-2 status as determined by real-time reverse transcription polymerase chain reaction (rRT-PCR) analysis of nasopharyngeal swabs were collected.

Delivery data, placing special focus on methods of delivery (vaginal or C-section), Apgar scores, and skin-to-skin contact were also collected.

All newborns were subjected to reviewing their history including gestational age, sex, birth weight, type of feeding, isolation, symptoms for up to 2 weeks of age, such as respiratory distress, oxygen desaturation, feeding problems, fever, and/or seizures, thorough clinical examination, review of laboratory evaluations, and rRT-PCR analysis of nasopharyngeal swab samples to determine SARS-CoV-2 status for those born to SARS-CoV-2-positive mothers.

\section{Statistical Analysis}

Data were verified, coded by the researcher and analyzed using IBM-SPSS $21{ }^{7}$ Data were expressed as mean, standard deviation, median, interquartile range (IQR), and percentages. Test of significances such as Chi-square and/or Fisher's exact tests were used to compare the difference in the distribution of frequencies among different groups. For continuous variables, independent $t$-test analysis was performed to compare the means of normally distributed data, while Mann-Whitney $U$-test was calculated to test the median differences of the data that do not follow a normal distribution. Significant baby-related variables from the univariate analysis were entered into a multivariable logistic regression model to test the independent baby-related correlates of SARS-CoV-2-positive mothers using odds ratio (OR), 95\% confidence interval (CI). A significant $p$-value was considered when it is less than 0.05 .

\section{Results}

A total of 79 mothers tested for SARS-CoV-2 were included in this study out of which 15 mothers (18.98\%) tested SARSCoV-2 positive.

As shown in -Table 1, the majority of mothers were African American for both groups. Maternal age ranged from 16 to 41 years (mean of 33.4 years for mothers in the SARS-CoV-2-positive group and 28.22 for mothers in the SARS-CoV-2-negative group). There were no significant differences between both groups regarding smoking, alcohol or drug use, comorbidities, group B streptococci (GBS), hepatitis B, HIV or rubella, or chorioamnionitis.

For all mothers, we found a significant association between symptoms and SARS-CoV-2 status; $40 \%$ of SARS-CoV2-positive mothers had fever, sore throat, cough, dyspnea without hypoxia, myalgias, or diarrhea at admission, whereas $60 \%$ of them were asymptomatic.

We found that 33 and $67 \%$ SARS-CoV-2-positive mothers delivered vaginally and via cesarean section, compared with 58 and 42\% SARS-CoV-2-negative mothers, respectively $(p=0.047)$. In addition, mean Apgar score at 5 minutes was 8.33 and 8.95 for newborns of SARS-CoV-2-positive and SARS-CoV-2-negative mothers, respectively. However, these values are of statistical significance alone and not of clinical significance.

Of note, we observed a significant association between both groups regarding skin-to-skin contact $(p<0.001)$; only 
1312 Characteristics of Newborns Born to SARS-CoV-2-Positive Mothers Farghaly et al.

\begin{tabular}{|c|c|c|c|}
\hline & $\begin{array}{l}\text { SARS-CoV-2 positive } \\
(n=15)\end{array}$ & $\begin{array}{l}\text { SARS-CoV-2 negative } \\
(n=64)\end{array}$ & $p$-Value \\
\hline \multicolumn{4}{|l|}{ Maternal demographics } \\
\hline Mother's age $(y)$ & $33.40 \pm 7.2$ & $28.22 \pm 6.8$ & $0.020^{\mathrm{a}}$ \\
\hline \multicolumn{4}{|l|}{ Race } \\
\hline African American & $11(73.4 \%)$ & $44(68.8 \%)$ & $0.749^{b}$ \\
\hline Hispanic & $2(13.3 \%)$ & $10(15.6 \%)$ & \\
\hline Others $^{\mathrm{e}}$ & $2(13.3 \%)$ & $10(15.6 \%)$ & \\
\hline Smoker & $0(0 \%)$ & $2(3.1 \%)$ & $0.654^{c}$ \\
\hline Alcohol abuse & $0(0 \%)$ & $2(3.1 \%)$ & $0.654^{c}$ \\
\hline Positive drug screen & $1(6.7 \%)$ & $8(12.5 \%)$ & $0.456^{c}$ \\
\hline Comorbidities & $6(40 \%)$ & $32(50 \%)$ & $0.342^{c}$ \\
\hline Contact with SARS-CoV-2 & $6(40 \%)$ & $4(6.3 \%)$ & $0.002^{c}$ \\
\hline \multicolumn{4}{|l|}{ Antenatal infections } \\
\hline RPR (syphilis) & $1(6.7 \%)$ & $0(0 \%)$ & $0.038^{c}$ \\
\hline Rubella (low/nonimmune) & $1(6.7 \%)$ & $4(6.3 \%)$ & $0.662^{c}$ \\
\hline Gonorrhea (positive) & $1(6.7 \%)$ & $0(0 \%)$ & $0.038^{c}$ \\
\hline GBS & $3(20 \%)$ & $12(18.8 \%)$ & $0.581^{\mathrm{b}}$ \\
\hline Chorioamnionitis & $1(6.7 \%)$ & $1(1.6 \%)$ & $0.257^{c}$ \\
\hline HbsAg (positive) & $0(0 \%)$ & $0(0 \%)$ & - \\
\hline HIV (positive) & $0(0 \%)$ & $0(0 \%)$ & - \\
\hline Chlamydia (positive) & $0(0 \%)$ & $0(0 \%)$ & - \\
\hline \multicolumn{4}{|l|}{ Maternal symptoms } \\
\hline Fever & $1(6.7 \%)$ & $3(4.7 \%)$ & $0.577^{c}$ \\
\hline Sore throat & $3(20 \%)$ & $0(0 \%)$ & $0.006^{c}$ \\
\hline Cough & $4(26.7 \%)$ & $0(0 \%)$ & $0.001^{c}$ \\
\hline Dyspnea & $2(13.3 \%)$ & $0(0 \%)$ & $0.034^{c}$ \\
\hline Hypoxia & $0(0 \%)$ & $0(0 \%)$ & - \\
\hline Myalgia & $3(20 \%)$ & $0(0 \%)$ & $0.006^{c}$ \\
\hline Diarrhea & $1(6.7 \%)$ & $0(0 \%)$ & $0.038^{c}$ \\
\hline Asymptomatic mothers & $9(60 \%)$ & $61(95.3 \%)$ & $0.001^{b}$ \\
\hline Mother's WBCs $\times 10^{9} / \mathrm{L}$ & $8.33 \pm 2.8$ & $10.03 \pm 2.9$ & $0.048^{\mathrm{a}}$ \\
\hline \multicolumn{4}{|l|}{ Delivery data } \\
\hline \multicolumn{4}{|l|}{ Method of delivery } \\
\hline Vaginal & $5(33 \%)$ & $37(58 \%)$ & $0.047^{\mathrm{b}}$ \\
\hline CS & $10(67 \%)$ & 27 (42\%) & \\
\hline Apgar score at 1 minute & $7.40 \pm 2.5$ & $8.58 \pm 0.9$ & $0.024^{\mathrm{a}}$ \\
\hline Apgar score at 5 minutes & $8.33 \pm 1.4$ & $8.95 \pm 0.3$ & $0.016^{\mathrm{a}}$ \\
\hline Skin-to-skin contact & $3(20 \%)$ & 49 (76.6\%) & $<0.001^{\mathrm{b}}$ \\
\hline Gestational age (wk) & $37.40 \pm 3.8$ & $38.67 \pm 1.8$ & $0.220^{\mathrm{a}}$ \\
\hline$\geq 37$ & $13(86.7 \%)$ & $61(95.4 \%)$ & $0.239^{c}$ \\
\hline$<37$ & $2(13.3 \%)$ & $3(4.6 \%)$ & \\
\hline Baby's sex (male/female) & $3 / 12$ & $28 / 36$ & $0.090^{\mathrm{b}}$ \\
\hline Birth weight $(\mathrm{kg})^{\mathrm{f}}$ & $2.8(1)$ & $3.1(0.5)$ & $0.230^{\mathrm{d}}$ \\
\hline
\end{tabular}

Abbreviations: CS, cesarean section; GBS, group B streptococci; HbsAg: hepatitis B surface antigen; RPR, rapid plasma reagin; SARS-CoV-2, severe acute respiratory syndrome-coronavirus-2; WBC, white blood cell.

andependent $t$-test was used to compare the means among groups.

${ }^{b}$ Chi-square analysis was used to compare the frequency among groups.

'Fisher's exact test was used to compare the frequency among groups.

${ }^{\mathrm{d}}$ Mann-Whitney U-test was used to compare the medians among groups.

eNative American, White, non-Hispanic/non-Latino, and others.

${ }^{\mathrm{f}}$ Median (interquartile range). 
Table 2 Newborn data by maternal SARS-CoV-2 status

\begin{tabular}{|c|c|c|c|}
\hline & $\begin{array}{l}\text { SARS-CoV-2 positive } \\
(n=15)\end{array}$ & $\begin{array}{l}\text { SARS-CoV-2 negative } \\
(n=64)\end{array}$ & $p$-Value \\
\hline Isolation & $11(73.3 \%)$ & $15(23.4 \%)$ & $<0.001^{a}$ \\
\hline Breast feeding & $5(33.3 \%)$ & $43(67.2 \%)$ & $0.016^{\mathrm{b}}$ \\
\hline NICU admission & $10(66.7 \%)$ & $16(25 \%$ & $0.003^{b}$ \\
\hline Nursery admission & $5(33.3 \%)$ & $48(75 \%)$ & \\
\hline \multicolumn{4}{|l|}{ Symptomatic babies } \\
\hline Respiratory distress & $6(42.9 \%)$ & 7 (10.9\%) & $0.010^{\mathrm{b}}$ \\
\hline Desaturation & $5(35.7 \%)$ & $4(6.3 \%)$ & $0.008^{b}$ \\
\hline Seizures & $1(6.7 \%)$ & $0(0 \%)$ & $0.038^{b}$ \\
\hline Poor feeding & $5(35.7 \%)$ & $4(6.3 \%)$ & $0.008^{b}$ \\
\hline Fever or hypothermia & $0(0 \%)$ & $0(0 \%)$ & - \\
\hline Asymptomatic babies & $5(33.3 \%)$ & $53(82.8 \%)$ & $<0.001^{\mathrm{a}}$ \\
\hline Abnormal baby's chest X-ray & $6(40 \%)$ & $4(6.3 \%)$ & $0.002^{b}$ \\
\hline Baby's 1 st WBCs $\times 10^{9} / \mathrm{L}$ & $13.89 \pm 1.2$ & $15.24 \pm 1.8$ & $0.593^{c}$ \\
\hline $\mathrm{CRP}(\mathrm{mg} / \mathrm{L})$ & $1.45 \pm 0.4$ & $1.10 \pm 0.3$ & $0.087^{c}$ \\
\hline Oxygen therapy & $7(46.7 \%)$ & $7(10.9 \%)$ & $0.004^{b}$ \\
\hline CPAP & $5(33.3 \%)$ & $5(7.8 \%)$ & $0.018^{b}$ \\
\hline Mechanical ventilation & $3(20 \%)$ & $1(1.6 \%)$ & $0.020^{\mathrm{b}}$ \\
\hline \multicolumn{4}{|l|}{ 2-week follow-up } \\
\hline Asymptomatic & 13 (86.7\%) & $0(100 \%)$ & \multirow[t]{2}{*}{$0.034^{\mathrm{b}}$} \\
\hline Symptomatic & $2(13.3 \%)$ & $0(0 \%)$ & \\
\hline
\end{tabular}

Abbreviations: CPAP, continuous positive airway pressure; CRP, C-reactive protein; NICU, neonatal intensive care unit; SARS-CoV-2, severe acute respiratory syndrome-coronavirus-2; WBC, white blood cell.

${ }^{a}$ Chi-square analysis was used to compare the frequency among groups.

bFisher's exact test was used to compare the frequency among groups.

'Independent $t$-test was used to compare the means among groups.

$20 \%$ of SARS-CoV-2-positive mothers had skin-to-skin contact with their babies, compared with $76.6 \%$ of SARS-CoV-2 -negative mothers.

Neonatal data were summarized in - Table 2. Both groups showed significant differences regarding isolation $(p<0.001)$; 73.3\% newborns from SARS-CoV-2-positive mothers were isolated, compared with $23.4 \%$ newborns from SARS-CoV-2negative mothers.

We found statistically significant differences for neonatal symptoms; $42.9 \%$ newborns of SARS-CoV-2-positive mothers had respiratory distress compared with only $10.9 \%$ newborns from SARS-CoV-2-negative mothers $(p=0.01)$. Likewise, $35.7 \%$ newborns of SARS-CoV-2 positive mothers had poor oral intake compared with only $6.3 \%$ newborns of SARS-CoV-2-negative mothers ( $p=0.008$ ).

There was no statistically significant association between initial white blood cells (WBCs) and C-reactive protein (CRP) for both groups ( $p=0.593$ and 0.087 , respectively). For newborns of SARS-CoV-2-positive mothers, the mean initial WBCs was $13.89 \times 10^{9} / \mathrm{L}$ and mean CRP was $1.45 \mathrm{mg} / \mathrm{L}$, whereas for newborns of SARS-CoV-2-negative mothers, the mean initial WBCs was $15.24 \times 10^{9} / \mathrm{L}$ and mean CRP was $1.10 \mathrm{mg} / \mathrm{L}$. On the other hand, there was a statistically significant difference for chest X-ray (CXR) between both groups; $40 \%$ newborns of SARS-CoV-2-positive mothers had abnormal CXR compared with only $6.3 \%$ newborns of SARSCoV-2-negative mothers $(p=0.002)$.

Interestingly, we had only one newborn who tested positive for SARS-CoV-2. A singleton full-term male was born to a SARS-CoV-2-positive 28-year-old asymptomatic mother by emergency cesarean section due to cord prolapse. The baby required resuscitation with positive pressure ventilation and intubation at birth. Apgar scores were 2, 4, and 5 at 1, 5, and 10 minutes, respectively. By the criteria of very low Apgar scores and poor tone, therapeutic total body hypothermia was provided for 72 hours in the NICU isolation room. Baby was on mechanical ventilation for 2 days and received ampicillin for 5 days and ceftazidime for 8 days. CXR on admission to NICU showed consolidation especially in the medial aspects of the left lower lobe which showed gradual clearing on subsequent CXRs on day 2 of life. Blood culture was negative. The first SARS-CoV-2 test resulted positive at the 25th hour of life. Repeated SARS-CoV-2 tests at 48 hours and 8 days of life were negative. CRP increased from $0.5 \mathrm{mg} /$ $\mathrm{dL}$ on day 1 to $1.5 \mathrm{mg} / \mathrm{dL}$ on day 2 . D-dimer showed a very high value at $809 \mathrm{ng} / \mathrm{mL}$ (reference range: $0-230 \mathrm{ng} / \mathrm{mL}$ ). Prothrombin time was 14.2 seconds, partial thromboplastin time was 36.2 seconds, and international normalized ratio 


\begin{tabular}{|lllll|}
\hline \multicolumn{2}{l}{ Table 3 Newborn characteristics correlated with SARS-CoV-2-positive mothers: logistic regression model } \\
\hline Variable & Univariate & & Multivariate \\
& OR $(95 \% \mathrm{CI})$ & $p$-Value & OR $(95 \% \mathrm{Cl})$ & $p$-Value \\
Skin-to-skin contact & $0.08(0.02-0.31)$ & $<0.001$ & $0.09(0.01-0.73)$ & 0.024 \\
Breast feeding & $0.24(0.07-0.81)$ & 0.021 & $0.35(0.04-2.77)$ & 0.318 \\
Isolation & $8.98(2.49-23.38)$ & 0.001 & $3.25(1.04-37.52)$ & 0.012 \\
NICU versus nursery admission & $6.0(1.78-11.19)$ & 0.004 & $10.2(3.66-21.33)$ & 0.014 \\
Respiratory distress & $6.11(1.64-12.81)$ & 0.007 & $2.19(0.77-9.22)$ & 0.041 \\
Desaturation & $8.33(1.88-16.97)$ & 0.005 & $2.83(1.58-15.65)$ & 0.027 \\
Poor feeding & $8.33(1.88-16.97)$ & 0.005 & $4.28(1.65-17.22)$ & 0.022 \\
Asymptomatic baby & $0.11(0.03-0.36)$ & $<0.001$ & $0.05(0.01-1.41)$ & 0.079 \\
Abnormal baby's chest X-ray & $10.0(2.36-23.45)$ & 0.002 & $7.93(2.89-22.89)$ & 0.027 \\
Oxygen therapy & $7.13(1.98-15.69)$ & 0.003 & $1.13(0.11-11.28)$ & 0.867 \\
CPAP & $5.90(1.44-14.15)$ & 0.014 & $1.90(0.01-12.39)$ & 0.312 \\
Mechanical ventilation & $15.75(1.51-26.47)$ & 0.021 & $5.19(2.09-17.16)$ & 0.044 \\
\hline
\end{tabular}

Abbreviations: $\mathrm{Cl}$, confidence interval; CPAP, continuous positive airway pressure; NICU, neonatal intensive care unit; OR, odds ratio; SARS-CoV-2, severe acute respiratory syndrome-coronavirus-2.

(INR) was 1.46 (reference range: 0.7-1.2). Head ultrasound, brain magnetic resonance imaging (MRI), and electroencephalogram (EEG) were all normal. Baby was discharged on the 12th day of life. Follow-up SARS-CoV-2 immunoglobulin (Ig)-M and IgG antibodies were negative at the 40th day of life.

All newborns were followed clinically until 2 weeks of age. All newborns of SARS-CoV-2 negative mothers were asymptomatic at their 2-week follow up. However, two newborns (13.3\%) of SARS-CoV-2-positive mothers were still symptomatic ( $p=0.034$ ) as they had poor feeding which improved later by the third week.

-Table 3 showed the multivariate regression analysis of the baby's correlates of SARS-CoV-2 positive mothers. The final multivariable logistic regression model contained seven independent correlates: skin to skin contact, newborn isolation, oxygen desaturation, poor feeding, abnormal baby's CXR, mechanical ventilation, and symptoms at the 2-week follow-up.

With this model, SARS-CoV-2-positive mothers were 90\% less likely to have skin-to-skin contact with their babies (adjusted odds ratio $[\mathrm{AOR}]=0.09,95 \% \mathrm{CI}$ : 0.01-0.73; $p=0.024)$. Furthermore, newborns of SARS-CoV-2-positive mothers were 3.3 times more likely to be isolated $(\mathrm{AOR}=3.25$, 95\% CI: $1.04-37.52 ; p=0.012$ ). Likewise, babies of SARS-CoV2-positive mothers were three times as likely to have desaturations in comparison to newborns from negative mothers (AOR $=2.83,95 \% \mathrm{CI}: 1.58-15.65 ; p=0.027)$. Also, newborns of SARS-CoV-2-positive mothers were four times more likely to have poor feeding compared with newborns of SARS-CoV-2negative mothers ( $\mathrm{AOR}=4.28,95 \% \mathrm{CI}: 1.65-17.22 ; p=0.022$ ).

Moreover, the likelihood of having abnormal CXR was about eight times higher in babies born to SARS-CoV-2positive mothers (AOR $=7.93, \quad 95 \%$ CI: 2.89-22.89; $p=0.027$ ). In addition, newborns of SARS-CoV-2-positive mothers were five times more likely to receive mechanical ventilation $(\mathrm{AOR}=5.19,95 \% \mathrm{CI}: 2.09-17.16 ; p=0.044)$. Finally, babies of SARS-CoV-2-positive mothers were 10 times more likely to be symptomatic at the 2-week follow-up, in comparison to newborns of SARS-CoV-2-negative mothers $(\mathrm{AOR}=10.20,95 \% \mathrm{CI}: 3.66-21.33 ; p=0.014)$.

\section{Discussion}

SARS-CoV-2 has led to a terrifying global pandemic. ${ }^{1,2}$ At BHMC, we serve a local community known to have many health disparities. We retrospectively collected and analyzed data of mothers tested for SARS-CoV-2 and their newborns. The majority of mothers were African American (74\%). Fifteen mothers (18.98\%) were SARS-CoV-2 positive. Only one newborn from a SARS-CoV-2-positive mother tested SARS-CoV-2 positive.

For mothers who tested SARS-CoV-2 positive, $40 \%$ of them had mild-to-moderate disease and $60 \%$ were asymptomatic. Maternal symptoms were mainly sore throat, cough, myalgias, fever, and diarrhea. Only six (40\%) mothers reported contact with suspected or confirmed cases of SARS-CoV-2. In this study, we found no association between maternal smoking, alcohol use, positive urine drug screen, comorbidities or antenatal infection, and maternal SARS-CoV-2 status.

Immediately after delivery, in our hospital, we avoided skinto-skin contact between the positive SARS-CoV-2 mothers and their babies. Eighty percent did not have skin-to-skin contact, while $20 \%$ had it as per parental shared decision, and for those newborns whose mothers were asymptomatic, they wore a mask and practiced good hand hygiene. Direct breastfeeding was avoided in SARS-CoV-2-positive mothers; only five newborns of positive mothers directly breastfed with precautions. However, the World Health Organization recommends that if a mother with confirmed or suspected SARS-CoV-2 chooses to directly breastfeed, she should wear a surgical mask and perform hand and breast hygiene prior to feeding. ${ }^{8}$ 
Regarding isolation, $73.3 \%$ of SARS-CoV-2-positive mothers were placed under droplet isolation, the other $26.7 \%$ did not have proper isolation due to maternal decision but were separated in an isolette. As per the recommendations of Centers for Disease Control and Prevention (CDC), the decision to separate newborns needs to be made on a case-by-case basis, using shared decision making between mothers and the health care team about the risks and benefits of it. ${ }^{9}$ We observed that although isolation measures in the motherbaby unit were not optimal, the newborns did not get infected.

Neonatal symptoms were mainly respiratory distress, desaturations, and poor feeding. We found statistically significant differences between newborns of SARS-CoV-2-positive and newborns of SARS-CoV-2-negative mothers regarding symptomatology, but no clinical significance, as shown in -Table 2. And this is in agreement with other studies that concluded that few newborns who tested positive soon after birth had dyspnea, fever, pneumonia, respiratory distress syndrome, and feeding intolerance, with overall mild disease and no deaths. ${ }^{4,10}$ Moreover, the likelihood of having abnormal CXR was about eight times higher in babies born to SARS-CoV-2-positive mothers. However, CXR finding was not specifically related to SARS-CoV-2, as it showed transient tachypnea of newborn (TTN) or respiratory distress syndrome (RDS). In addition, newborns of SARS-CoV-2 positive mothers were five times more likely to receive mechanical ventilation. However, we believe this is only a statistical significance as these newborns had other comorbidities such as TTN, RDS, and prematurity, but we were unable to stratify due to the small sample size.

All newborns were asymptomatic at 2 weeks of age except for two newborns of SARS-CoV-2-positive mothers who were still in NICU. However, these two babies tested three times SARS-CoV-2 negative and they had other problems like prematurity and RDS. At discharge, we gave all mothers advice regarding home isolation precautions as guidelines from the American Academy of Pediatrics. Infants were followed-up clinically via telehealth and by a repeat test for SARS-CoV-2 2 days after discharge, and then later at 1 to 2 weeks after discharge.

Interestingly, in our study, only one newborn tested positive for SARS-CoV-2. He was born by emergency cesarean section due to cord prolapse, had low Apgar scores, and required intubation in the delivery room. Although perinatal asphyxia cannot be excluded as a cause of illness, the pulmonary consolidation found in CXR, a positive SARSCoV-2 PCR test, rising CRP, and very high D-dimer made neonatal SARS-CoV-2 infection a strong differential diagnosis for this critical illness. Furthermore, the mother wore a face mask throughout the hospital stay, had no skin-to-skin contact with the baby, no direct breastfeeding, no visitors (including parents) were allowed during the newborn's first 5 days of life, and strict droplet isolation precautions took place. Despite these precautions, the newborn tested positive for SARS-CoV-2 at 25 hours of life. These findings together with the CXR raised our concern about a possible vertical transmission. However, horizontal contamination is still a possibility as the baby tested negative for SARS-CoV-2
PCR at 48 hours and at 8 days of life, and his SARS-CoV-2 IgG and IgM antibodies were also negative.

\section{Limitations}

Although Mullins et al concluded that $50 \%$ of the mothers with SARS-CoV-2 delivered preterm infants, ${ }^{11}$ a limitation to our study was the small number of preterm newborns. Most newborns were full term, only $13.3 \%$ of newborns from SARS-CoV-2-positive mothers were preterm, so we could not stratify according to the gestational age. However, prematurity is expected as obstetricians tend to deliver SARS-CoV-2positive mothers sooner before their clinical condition worsens. Another limitation was the small sample size which would need a longer enrollment period or a multicenter study.

\section{Conclusion}

In conclusion, SARS-CoV-2 has caused major morbidity and mortality worldwide. Neonates born to mothers with confirmed or suspected SARS-CoV-2 are most of the time asymptomatic. However, neonatal critical illness is still a possibility; therefore, isolation precautions (such as avoiding skin-to-skin and direct breastfeeding) and vertical transmission should be studied thoroughly. In addition, testing these newborns by nasopharyngeal swab at least at 24 hours after birth and monitoring them for the development of symptoms for 14 days after birth are needed.

Authors' Contributions

M.A.A.F., F.K., and R.M.K. designed the study. M.A.A.F. and F.C. collected the data and performed the data analysis. F.K. and R.M.K. revised the data analysis, edited the manuscript, and critically revised the final version. F.C. submitted the manuscript. All authors read and approved all the manuscript.

Funding

None.

Conflict of Interest

None declared.

Acknowledgments

The authors thank the staff in the Neonatal Intensive Care Unit (NICU) and Mother-Baby Unit (Nursery) at BHMC in New York City.

\section{References}

1 World Health Organization. Coronavirus disease 2019(SARS-CoV-2). Situation Report-74. Available at: https://www.who.int/docs/default-source/coronaviruse/situation-reports/20200403-sitrep-74covid-19-mp.pdf?sfvrsn=4e043d03_14. Accessed August 6, 2020

2 Coronaviridae Study Group of the International Committee on Taxonomy of Viruses. The species severe acute respiratory syndrome related coronavirus: classifying 2019-nCoV and naming it SARSCoV-2. Nat Microbiol 2020;5:536-544

3 Chen H, Guo J, Wang C, et al. Clinical characteristics and intrauterine vertical transmission potential of COVID-19 infection in 
nine pregnant women: a retrospective review of medical records. Lancet 2020;395(10226):809-815

4 Alzamora MC, Paredes T, Caceres D, Webb CM, Valdez LM, La Rosa M. Severe COVID-19 during pregnancy and possible vertical transmission. Am J Perinatol 2020;37(08):861-865

5 Centers for Disease Control and Prevention. Coronavirus disease 2019 in children-United States, February 12-April 2, 2020. Morbidity and Mortality Weekly Report 2020;69(14):422-426

6 Chandrasekharan P, Vento M, Trevisanuto D, et al. Neonatal resuscitation and postresuscitation care of infants born to mothers with suspected or confirmed SARS-CoV-2 infection. Am J Perinatol 2020;37(08):813-824

7 IBM-SPSS. Statistical Package for Social Science.Ver.21. Available at: https://www.ibm.com/support/pages/spss-statistics-210available-download. Accessed August 6, 2020
8 World Health Organization. Breastfeeding and COVID-19. Available at: https://www.who.int/news-room/commentaries/detail/ breastfeeding-and-covid-19. Accessed August 6, 2020

9 Centers for Disease Control. Considerations for inpatient obstetric healthcare settings. Available at: https://www.cdc.gov/coronavirus/2019-ncov/hcp/inpatient-obstetric-healthcare-guidance.html. Accessed August 6, 2020

10 Chen Rong, Zhang Yuan, Huang Lei, Cheng Bi-heng, Xia Zhongyuan, Meng Qing-tao. Safety and efficacy of different anesthetic regimens for parturients with SARS-CoV-2 undergoing cesarean delivery: a case series of 17 patients (in French). Can J Anaesth 2020 (e-pub ahead of print) . Doi: 10.1007/s12630-020-01630-7

11 Mullins E, Evans D, Viner RM, O'Brien P, Morris E. Coronavirus in pregnancy and delivery: rapid review. Ultrasound Obstet Gynecol 2020;55(05):586-592 